WellBeing International

WBI Studies Repository

9-2011

\title{
Indicators of Age, Body Size and Sex in Goat Kid Calls Revealed Using the Source-Filter Theory
}

Elodie F. Briefer

Queen Mary University of London

Alan G. McElligott

Queen Mary University of London

Follow this and additional works at: https://www.wellbeingintlstudiesrepository.org/acwp_asie

Part of the Animal Studies Commons, Comparative Psychology Commons, and the Other Animal

Sciences Commons

\section{Recommended Citation}

Briefer, E., \& McElligott, A. G. (2011). Indicators of age, body size and sex in goat kid calls revealed using the source-filter theory. Applied Animal Behaviour Science, 133(3), 175-185.

This material is brought to you for free and open access by WellBeing International. It has been accepted for inclusion by an authorized administrator of the WBI Studies Repository. For more information, please contact wbisr-info@wellbeingintl.org.

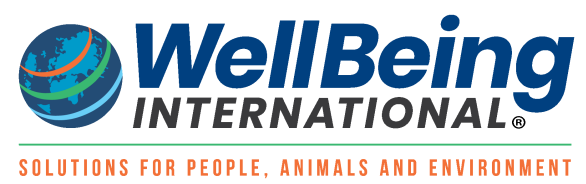




\title{
Indicators of Age, Body Size and Sex in Goat Kid Calls Revealed Using the Source-Filter Theory
}

\author{
Elodie Briefer, Alan G. McElligott \\ Queen Mary University of London
}

\section{KEYWORDS:}

acoustic analysis, Capra hircus, ungulate, vocal communication, vocal development

\begin{abstract}
:
The source-filter theory is an important framework recently applied to the study of animal vocalisations, which links the mode of vocal production to call parameters. Vocalisations can be good indicators of a sender's characteristics, such as identity, body size, age, and even hormonal status and affective states. For these reasons, applied vocal communication research would greatly benefit from adopting the source-filter theory approach to identify key call parameters linked to physical and physiological characteristics of domestic animals. Here, we introduce the source-filter theory through a detailed analysis and interpretation of goat contact calls during development. In mammals, vocal development is mostly influenced by maturation. Maturational processes of vocalisations are linked to growth or sex hormone effects on the larynx and vocal tract. We investigated changes to the parameters of goat contact calls during ontogeny, according to age, body size and sex. We recorded goat kids from birth to four months old and analysed their calls by considering the shape and functioning of the vocal apparatus. We found age and sex-related changes to most of the measured vocal parameters, suggesting a direct or indirect effect of sex hormones on vocal ontogeny. Furthermore, body size growth was negatively correlated with most frequency parameters, indicating that vocal production is constrained by body size throughout development. Therefore kid vocalisations provide information about age, sex and body size. We suggest that similar analyses applied to the study of vocal correlates of affective states, could greatly help the discovery of convenient and non-invasive indicators of animal welfare.
\end{abstract}

\section{Introduction}

According to the source-filter theory of voice production (Fant, 1960), mammal vocalisations are generated by vibrations of the vocal folds ("source") and are subsequently filtered by the vocaltract("filter"). This framework, originally developed to study human speech (Titze, 1994), has recently proved very useful for animal vocal communication research by linking vocal production mechanisms to call parameters (Taylor and Reby, 2010). Source-related vocal parameters depend on the anatomy/physiology ofthe larynx and the sub-laryngeal and laryngeal structures (e.g. vocal fold length and mass), whereas filter-related vocal parameters are determined by the anatomy/physiology of the supralaryngeal vocal tract (e.g. shape and length of the vocal tract). We suggest that this framework could greatly benefit applied vocal communication research by identifying key vocal parameters and link them to the structures of the vocal apparatus of domestic animals. This would allow a far better 
understanding of why and to what extent call structure varies between individuals and between tested situations.

The development of animal vocalisations is influenced by growth, maturation and experience. Mammal vocalisations, with few exceptions (Janik and Slater, 1997), are believed to be genetically determined with only limited flexibility (Seyfarth and Cheney, 2010). Changes during call ontogeny are mainly attributed to maturational processes. However, developmental changes in vocal production (acoustic parameters of vocalisations) have mostly been investigated in mammals with complex vocal repertoires (e.g. bats, Jones and Ransome, 1993; meerkats, Suricata suricatta, Hollén et al., 2008; primates, Ey et al., 2007a; seals, Khan and Markowitz, 2006). Other mammals with less complex repertoires have received little attention, and knowledge of the influence of factors such as growth on vocal parameters is weak (e.g. felids, Peters, 2011).

Changes in vocalisations during development can be linked to growth and sex-related hormonal changes. The larynx is a steroid receptor target organ (Aufdemorte et al., 1993), and therefore, source-related vocal parameters can be affected during development by changes in testosterone levels or by growthrelated lengthening of the vocal folds (Taylor and Reby, 2010). The vocal tract grows with the rest of the body and its length is directly dependent on body size (Taylor and Reby, 2010). Because of the negative relationship between frequency parameters and body size (or weight) among adult individuals in several species (Reby and McComb, 2003; Vannoni and McElligott, 2008), the same negative correlation is expected for juveniles during growth (Peters, 2011). Filter-related vocal parameters should thus change during development, with body sizerelated lengthening and widening of the vocal tract.

The physical and physiological characteristics of animals are strongly linked to the vocalisations they produce (Taylor and Reby, 2010). For these reasons, various vocal parameters have proven to be good indicators of caller identity (Vannoni and McElligott, 2007; Briefer and McElligott, in press), quality (Reby et al., 2005; Briefer et al., 2010) and even hormonal status (Charlton et al., 2010). Vocalisations could also be reliable indicators of inner states (Gogoleva et al., 2010). Indeed, emotions are associated with physiological and neurological changes that affect respiration, salivation and tension of the vocal folds and vocal tract walls, causing modifications of the vocal parameters (Scherer, 1986). Therefore vocal parameters are promising non-invasive tools to assess animal welfare (Weary and Fraser, 1995; Manteuffel et al., 2004). However, in order to find vocal indicators of affective states, a good understanding of the vocal production mechanisms and the way emotions influence vocal production is required. More importantly, a thorough knowledge of the structure of vocalisations produced in low-affect situations (e.g. contact calls produced at close distance) at every step of vocal ontogeny is crucial in order to detect the subtle variations in structure that indicate different inner states. In this study, we introduce the source-filter theory through a detailed analysis and interpretation of goat contact calls during ontogeny.

We investigated changes during development in vocal characteristics according to age, body size and sex in a highly vocal livestock species, the goat (Capra hircus). In this highly social and vocal species, an exclusive bonding between the mother and the neonate is developed within a few hours postpartum (Poindron et al., 1993; Miranda-de la Lama and Mattiello, 2010). As a consequence, mutual mother-kid vocal recognition is evident from the first week postpartum, and this recognition probably relies on the individual characteristics of the calls (Briefer and McElligott, in press). Here, we studied age-related changes according to sex in both source- and filter-related vocal parameters, as well as intensity parameters, from birth to four months of age. We also investigate body size-related changes in vocalisations. We predicted that most vocal parameters would change with age as a result of body growth, and that these changes would be sex-dependent. 


\section{Methods}

\subsection{Subjects and management conditions}

The study was carried out at White Post Farm, Nottinghamshire $\left(53^{\circ} 06^{\prime} \mathrm{N}, 1^{\circ} 03 \mathrm{~W}\right)$, UK, on four groups of $\left.1^{\circ} 03^{\prime} \mathrm{W}\right)$, UK, on four groups of pygmy goat kids born in July 2009, December 2009, March 2010 and July 2010 , respectively ( $n=23 ; 5$ females and 18 males; mean \pm SE group size $=5.8 \pm 0.5$ kids). All kids had the same father (i.e. they were either full or half siblings) and consisted of four single born, eight pairs of twins and one set of triplets.

The goats used in this study were kept indoors in a communal pen of $4.4 \mathrm{~m} \times 4.5 \mathrm{~m}$. When a female was about to give birth, she was isolated in a $2.5 \mathrm{~m} 2$ pen within the communal pen and kept there with her kid(s) for 2-3 days to allow adequate development of the mother-offspring relationship and prevent interference from other goats. Mothers and kids were then released in the communal pen with the other goats. At 2-3 months postpartum, all the goats (mothers and kids together) were moved to a bigger pen with other individuals. Male kids were castrated by using a constrictive rubber ring applied to the neck of the scrotum 3-4 days after birth (usual procedure in UK; DEFRA, 2009). This was carried out as part of routine management of the animals by the owners.

\subsection{Recordings and selection of calls}

Goats produce two kinds of calls when isolated, according to the degree of stress of the situation. "Contact calls" are the most common, and are used to maintain contact with other goats when physically, but not vocally separated, whereas "distress calls" are high-pitched, and associated with high stress levels (Lenhardt, 1977). In other species, distress calls have been shown to generally lack useful information such as individual identity, or at least to contain less information than contact calls (e.g. red deer, Cervus elaphus,Vanková andMálek, 1997; chacma baboons, Papio ursinus, Rendall et al., 2009). Because we were interested in low-affect vocalisations potentially encoding information about age, body size and sex, we focussed in this study on contact calls. We recorded goat kid contact calls at distances of $1-5 \mathrm{~m}$ from the vocalising animal using a Sennheiser MKH70 directional microphone, connected to a Marantz PMD660 numeric recorder (sampling rate: $44.1 \mathrm{kHz}$ ) between 10:00 and 17:00 $\mathrm{h}$. Kids were recorded daily during their first week (0-10 days old), at approximately 5 weeks old (27-41 days old), and at approximately 3 or 4 months old (104-124 days old) for four kids (one female and three males) that had been kept at the farm with their mothers (days of recording per individual, mean \pm SE $=9.70 \pm 0.38$, range $=6-14)$. This was done by separating kids during no more than $5 \mathrm{~min}, 2-3$ times per day at visual and hearing range $(1-10 \mathrm{~m})$ from their mothers. The distance to the mother was set at $1 \mathrm{~m}$ on average during the first day of recordings and increased afterwards if necessary, until we obtained contact calls (i.e. low-affect vocalisations) and no distress calls (see Supplementary Material). Kids were isolated alone, except if they were stressed during isolation even at $1 \mathrm{~m}$. In these cases, they were isolated with their sibling(s).

Vocalisations were imported into a computer at a sampling rate of $44.1 \mathrm{kHz}$ and saved in WAV format at 16-bit amplitude resolution. We used Praat v.5.0.47 DSP Package (Boersma and Weenink, 2009) and Seewave (Sueur et al., 2008) for subsequent analyses. Calls were visualised on spectrograms in Praat(FFT method, window length $=0.01 \mathrm{~s}$, time steps $=1000$, frequency steps $=250$, Gaussian window shape, dynamic range $=50 \mathrm{~dB}$ ).

To assess age, sex and body size effects on kid calls, we selected eight good quality calls (low levels of background noise as visualised on a spectrogram) per kid per day for subsequent analyses (total $=1784$ calls from 23 kids, mean \pm SE per kid $=77.58 \pm 3.04$ calls). Selected calls were separated by at least 
three calls within a given recorded bout. This is important in order to avoid pseudoreplication, because consecutive calls are more likely to be homogeneous (Reby et al., 1999). We strongly recommend this approach for other studies of vocalisations.

\subsection{Acoustical analysis}

Goat calls are short, with a clear harmonic structure and strong frequency and amplitude modulations (Fig. 1). We extracted source-related (fundamental frequency, " $F$ ) and filter-related (amplified frequencies, "formants") vocal parameters as well as intensity measures using a custom built program in Praat. This program batch processed the editing, the setting of parameters, the analyses and the exporting of output data (Reby and McComb, 2003; Charlton et al., 2009a). The vocal parameters that we measured are listed in Table 1 and detailed in the next paragraphs below (Praat commands are indicated in brackets). Goats produce two kinds of contact calls: closed-mouth contact calls and open-mouth contact calls (RuizMiranda et al., 1993). The extent of mouth opening influences the resonant properties of the vocal tract (Titze, 1994). Several vocal parameters thus differ between closed- and open-mouth calls (Sèbe et al., 2010), especially the frequencies and amplitude levels of formants. Because some formants are suppressed in closed-mouth calls, only kid contact calls emitted with the mouth open, in which formants are more evident, were used in this study.

Source-related vocal parameters were measured by extracting the F0 contour of each call using a crosscorrelation method ([Sound: To Pitch (cc) command], 0-10 days old: time step $=0.005 \mathrm{~s}$, pitch floor $=$ $300-400 \mathrm{~Hz}$, pitch ceiling $=700-900 \mathrm{~Hz} ; 27-41$ days old: time step $=0.005-0.015 \mathrm{~s}$, pitch floor $=200-$ $300 \mathrm{~Hz}$, pitch ceiling $=700-800 \mathrm{~Hz} ; 104-124$ days old: time step $=0.015 \mathrm{~s}$, pitch floor $=100-200 \mathrm{~Hz}$, pitch ceiling $=600-800 \mathrm{~Hz}$ ). If the entire F0 contour could not be detected by the cross-correlation method, calls were high-pass filtered before the analysis (cut-off frequency: 0-10 days old, $300 \mathrm{~Hz}$; $27-$ 41 days old, $200 \mathrm{~Hz} ; 104-124$ days old, $100 \mathrm{~Hz}$ ). The frequency value of F0 at the start (F0Start) and at the end (FOEnd) of the call; the mean (FOMean), minimum (FOMin) and maximum (FOMax) F0 frequency values across the call; the percentage of the total call duration when FO was maximum (TimeFOMax); and the FO mean absolute slope (FOAbsSlope) were included in our analyses. To characterise F0 variation along the call, we measured the mean F0 variation per second (FOvar), calculated as the cumulative variation in the FO contour in Hertz divided by call duration. We also measured the number of complete cycles of F0 modulation per second (FMRate) and the mean peak-to-peak variation of each F0 modulation (FMExtent, see Charlton et al., 2009a). Finally, we included two other measures of F0 variation: jitter (Jitter, Titze et al., 1987), which is the mean absolute difference between frequencies of consecutive FO periods divided by the mean frequency of FO [Jitter (local) command]; and shimmer (Shimmer), which is the mean absolute difference between the amplitudes of consecutive F0 periods divided by the mean amplitude of FO [Shimmer (local) command].

Filter-related (formants) vocal parameters were measured by extracting the contour of the first four formants of each call using Linear Predictive Coding analysis (LPC [Sound: To Formant (burg) command], $0-10$ days old: time step $=0.003 \mathrm{~s}$, maximum number of formants $=4-5$, maximum formant $=$ 9800-12,000 Hz, window length $=0.01-0.04 \mathrm{~s} ; 27-41$ days old: time step $=0.01-0.025 \mathrm{~s}$, maximum number of formants $=4-5$, maximum formant $=8000-10,000 \mathrm{~Hz}$, window length $=0.01-0.05 \mathrm{~s} ; 104-124$ days old: time step $=0.025 \mathrm{~s}$, maximum number of formants $=4-5$, maximum formant $=5000-7000 \mathrm{~Hz}$, window length $=0.05 \mathrm{~s}$ ). We compared the outputs of the LPC analysis with visual inspections of spectrograms to check if Praat accurately tracked the formants. Spurious values and inter-segment values (see Fig. 1) were deleted and we corrected for octave jumps when necessary. We included in our analyses the mean (F1-4Mean) minimum (F1-4Min), and maximum (F1-4Max) values of the formants. We then estimated the minimum spacing of the formants (DfMin), known as minimum formant dispersion, using the method described by Reby and McComb (2003). Additionally, we included in our analyses the 
frequency values at the upper limit of the first (Q25\%), second (Q50\%) and third (Q75\%) quartiles of energy, measured on a linear amplitude spectrum applied to the whole call.

We measured intensity characteristics by extracting the intensity contour of each call [Sound: To Intensity command]. We then included in our analyses the mean variation per second (AmpVar), calculated as the cumulative variation in amplitude divided by the total call duration; the number of complete cycles of amplitude modulation per second (AMRate); and the mean peak-to-peak variation of each amplitude modulation (AMExtent, see Charlton et al., 2009a).

We also included in our analyses the total duration of each call (Dur). Because calls of 0- to 10-day-old kids are relatively short and composed of short segments (Fig. 1), FOvar, FMRate, FMExtent, AMVar, AMRate and AMExtend were only measured for older kids (total number of parameters included in the analyses: 26 parameters for 0 - to 10-day-old kid calls and 32 parameters for 27- to 124-day-old kid calls). Some parameters could not be measured in every call, resulting in a small proportion of missing values $(0.63 \%$ of the values). Therefore, the sample size (number of calls) differs between the vocal parameters.
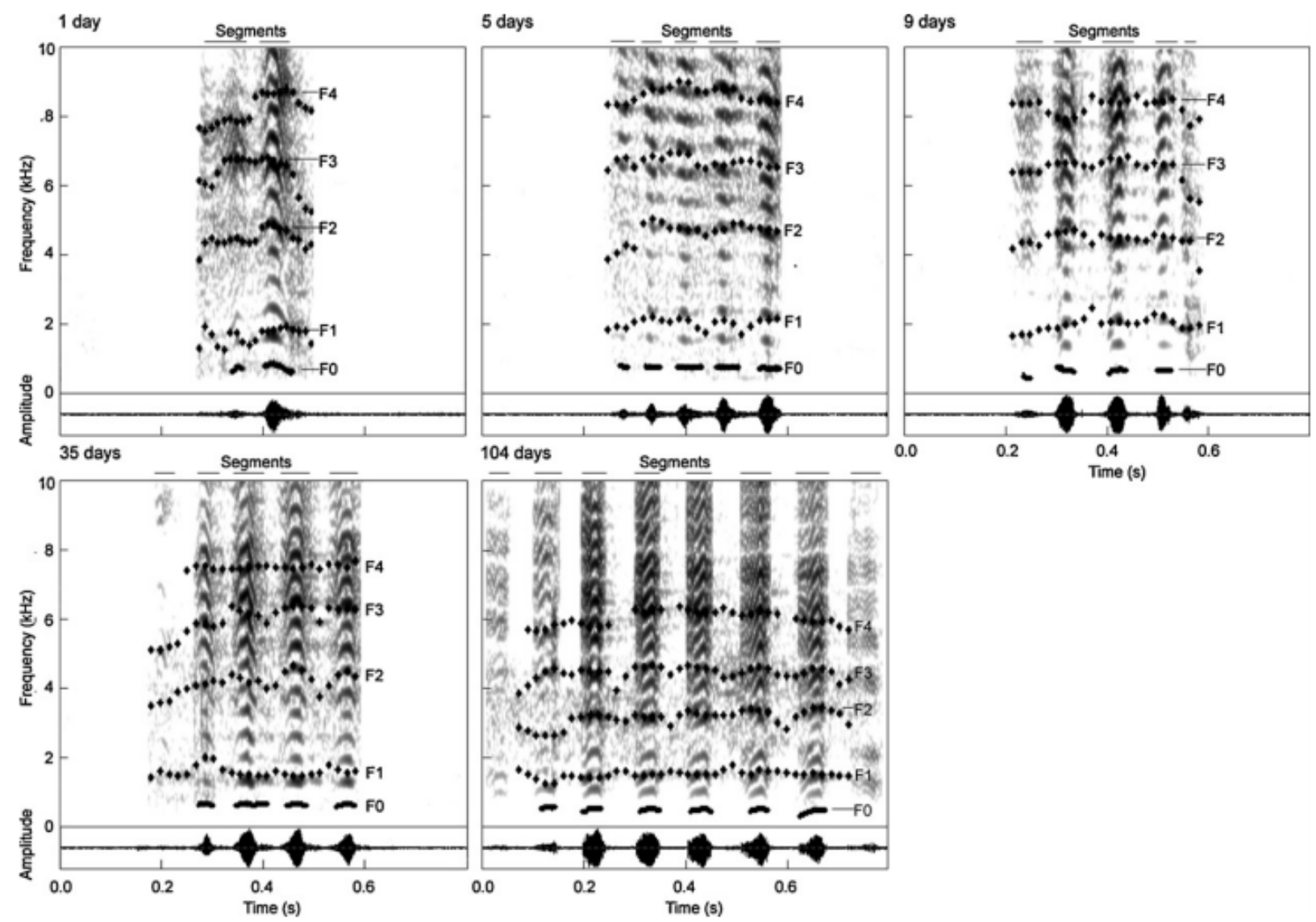

Fig. 1. Spectrograms (above) and oscillograms (below) of one call produced by the same female kid at 1 day, 5 days, 9 days, 35 days and 104 days. The black line at the bottom indicates the fundamental frequency (F0) and black dots above indicate the frequency values of the first four formants (F1-4). Calls are composed of small acoustic units ("segments"), which are indicated above the spectrograms. Only formant values of the segments were kept for the analyses. 


\subsection{Size measurements}

Body size was assessed using a proxy indicator of skeletal size. A segment of the left hind leg, from the tip of the dew claw to the top of the hock was measured on several of the recording days for each kid ( $28.70 \%$ of the days, $2.78 \pm 0.23$ days per kid). This measurement was chosen because it is less prone to error than others, and has been used as an indicator of body size for other ungulates such as fallow deer (Dama dama, McElligott et al., 2001) and sheep (Ovis aries, Coltman et al., 1999).

\subsection{Statistical analysis}

We used Linear Mixed Models (LMMs) fitted with Restricted Estimate Maximum Likelihood (REML, Ime function in R, Venables and Ripley, 2001) to investigate the effects of age (range:0-124days old, continuous predictor), sex (male/female, categorical predictor) and body size (leg measurement, range: 8-11.5 cm, continuous predictor) on the vocal parameters of kid calls. We carried out a first series of models (one for each vocal parameter) to investigate the effect of age on vocal parameter measures. In these models, we included the vocal parameter as a dependent variable and the age of kids as a fixed effect. Then, to investigate if some of the age effects were due to body size changes, we carried out a second series of models (one for each vocal parameter), in which we examined the effect of body size (leg measurements) on the mean values for each vocal parameter per kid per day (from calls recorded on the same days as when kid leg size had been measured). In these models, the vocal parameter was included as a dependent variable, and we included as a fixed effect the size of the hind leg of the kids.

In both series of models, to investigate sex effects, independently of age and body size, respectively, we included sex as a second fixed effect. In the first series of models (age), the interaction effect between age and sex was also included. The identity of kids nested within the identity of their mothers was fitted as a random term in both series of models to account for individual and kinship (full or half siblings) differences. To summarise, the first series of models included age, sex and the interaction between age and sex as fixed effects, as well as the identity of kids nested within the identity of their mothers as a random term. The second series included body size and sex as fixed effects, as well as the identity of kids nested within the identity of their mothers as a random term.

Q-Q plots and scatterplots of the residuals of the dependent variables were inspected visually to ensure their normal distribution, and data were log-transformed when necessary. The calculation of a coefficient of determination R2 for GLMM is not obvious because of the presence of random effects. We thus estimated R2 following (Magee, 1990) to describe the way models fitted the observed data, as follows: R2 $=1-\exp (-2 / n(\log L M-\log L 0))$, where $n$ is the number of observations (calls), log LM is the standard log-likelihood of the model (which include fixed and random effects) and log LO is the standard loglikelihood of the intercept-only model. We carried out statistical analyses using R v.2.9.0 (R Development Core Team, 2009). All tests were two tailed and factors were considered to have a statistically significant influence if $p<0.05$. All means are given with SEs.

\subsection{Ethics}

Animal care and all experimental procedures were in accordance with the International Society for Applied Ethology guidelines. During the recordings, mothers and kids could always see each other and were separated for no more than $5 \mathrm{~min}$ at $1-10 \mathrm{~m}$. 
Table 1. Values of the vocal parameters measured for males and females at each age period (0-10 days, 27-41 days and 104-124 days; mean (bold) \pm SE). See Section 2 for abbreviation of the vocal parameters.

\begin{tabular}{|c|c|c|c|c|c|c|c|c|c|c|c|c|}
\hline \multirow[t]{3}{*}{ Parameter } & \multicolumn{4}{|c|}{$0-10$ days } & \multicolumn{4}{|c|}{ 27-41 days } & \multicolumn{4}{|c|}{ 104-124 days } \\
\hline & \multicolumn{2}{|l|}{ Males } & \multicolumn{2}{|l|}{ Females } & \multicolumn{2}{|l|}{ Males } & \multicolumn{2}{|l|}{ Females } & \multicolumn{2}{|l|}{ Males } & \multicolumn{2}{|l|}{ Females } \\
\hline & Mean & SE & Mean & SE & Mean & SE & Mean & SE & Mean & SE & Mean & SE \\
\hline F0Start (Hz) & 591.8 & 3.3 & 582.1 & 5.2 & 537.1 & 3.5 & 535.1 & 4.6 & 432.9 & 13.6 & 478.7 & 3.3 \\
\hline F0End $(\mathrm{Hz})$ & 590.4 & 2.8 & 547.8 & 4.6 & 502.9 & 3.5 & 495.9 & 4.7 & 406.5 & 15.6 & 446.9 & 11.9 \\
\hline FOMean $(\mathrm{Hz})$ & 606.6 & 2.7 & 591.4 & 4.2 & 536.6 & 3.1 & 551.3 & 3.1 & 439.9 & 12.0 & 443.8 & 10.3 \\
\hline F0Min $(\mathrm{Hz})$ & 563.2 & 3.0 & 523.6 & 4.5 & 488.3 & 3.3 & 485.5 & 4.3 & 383.4 & 12.1 & 443.8 & 10.3 \\
\hline F0Max $(\mathrm{Hz})$ & 634.1 & 2.7 & 634.0 & 4.3 & 565.4 & 3.1 & 580.8 & 2.8 & 467.3 & 12.9 & 491.1 & 3.1 \\
\hline TimeF0Max (\%) & 43.2 & 0.8 & 38.4 & 1.4 & 36.6 & 1.4 & 40.8 & 1.8 & 45.2 & 5.8 & 37.7 & 4.4 \\
\hline F0AbsSlope (Hz/s) & 510.5 & 11.7 & 680.4 & 21.1 & 330.9 & 11.8 & 393.9 & 17.8 & 262.5 & 27.1 & 166.0 & 18.0 \\
\hline FOVar (Hz/s) & N/A & N/A & N/A & $N / A$ & 212.0 & 6.7 & 298.3 & 15.6 & 170.3 & 25.2 & 108.8 & 20.3 \\
\hline FMRate $\left(s^{-1}\right)$ & N/A & N/A & N/A & N/A & 4.1 & 0.1 & 5.7 & 0.2 & 4.1 & 0.3 & 5.5 & 0.7 \\
\hline FMExtent $(\mathrm{Hz})$ & N/A & N/A & N/A & N/A & 66.3 & 3.4 & 57.9 & 3.7 & 39.7 & 5.2 & 20.0 & 2.7 \\
\hline Jitter (\%) & 4.2 & 0.1 & 3.4 & 0.1 & 4.3 & 0.1 & 2.3 & 0.1 & 4.1 & 0.4 & 2.8 & 0.5 \\
\hline Shimmer (\%) & 18.3 & 0.2 & 14.6 & 0.3 & 17.9 & 0.2 & 13.9 & 0.3 & 16.7 & 0.7 & 18.4 & 0.7 \\
\hline F1Mean $(\mathrm{Hz})$ & 1863.6 & 12.7 & 1986.8 & 22.0 & 1693.1 & 10.6 & 1717.3 & 17.7 & 1284.5 & 29.6 & 1433.4 & 42.4 \\
\hline F2Mean $(\mathrm{Hz})$ & 4211.8 & 27.8 & 4508.3 & 47.7 & 3880.9 & 23.0 & 3736.3 & 39.8 & 2948.9 & 77.3 & 2822.2 & 50.7 \\
\hline F3Mean $(\mathrm{Hz})$ & 6496.8 & 16.8 & 6801.0 & 34.6 & 5686.9 & 24.5 & 5739.2 & 29.5 & 4323.9 & 46.9 & 4544.0 & 48.1 \\
\hline F4Mean $(\mathrm{Hz})$ & 8483.5 & 19.2 & 8778.4 & 35.0 & 7564.6 & 30.6 & 7601.5 & 32.4 & 5711.4 & 60.5 & 5876.7 & 79.7 \\
\hline F1Min $(\mathrm{Hz})$ & 1445.2 & 14.8 & 1594.1 & 24.8 & 1369.1 & 14.7 & 1349.1 & 24.1 & 1048.7 & 48.1 & 1215.6 & 58.6 \\
\hline F2Min $(\mathrm{Hz})$ & 3715.2 & 28.8 & 4081.2 & 52.5 & 3485.2 & 28.0 & 3353.9 & 47.6 & 2624.2 & 74.9 & 2531.2 & 52.2 \\
\hline F3Min $(\mathrm{Hz})$ & 6069.5 & 18.7 & 6441.3 & 36.9 & 5254.1 & 25.2 & 5296.9 & 30.6 & 3914.0 & 64.0 & 4163.2 & 100.3 \\
\hline F4Min $(\mathrm{Hz})$ & 8033.2 & 20.9 & 8366.7 & 38.1 & 7094.6 & 31.4 & 7204.2 & 38.1 & 5321.5 & 71.9 & 5648.2 & 88.1 \\
\hline F1Max (Hz) & 2229.6 & 15.5 & 2303.6 & 26.0 & 1986.1 & 16.1 & 1997.0 & 21.7 & 1520.2 & 34.3 & 1564.7 & 69.1 \\
\hline F2Max $(\mathrm{Hz})$ & 4674.1 & 28.3 & 4899.8 & 47.2 & 4255.1 & 24.9 & 4096.0 & 40.5 & 3251.6 & 83.0 & 3079.8 & 85.4 \\
\hline F3Max $(\mathrm{Hz})$ & 6913.4 & 19.0 & 7158.1 & 37.6 & 6154.7 & 30.9 & 6164.7 & 35.5 & 4753.0 & 80.4 & 4935.8 & 44.5 \\
\hline F4Max (Hz) & 8972.8 & 21.2 & 9255.0 & 37.5 & 8072.0 & 36.2 & 8030.6 & 35.3 & 6096.8 & 79.5 & 6137.1 & 75.1 \\
\hline DfMin $(\mathrm{Hz})$ & 2215.1 & 7.9 & 2268.5 & 11.9 & 1943.3 & 9.5 & 1965.6 & 11.7 & 1457.2 & 20.2 & 1505.2 & 21.0 \\
\hline Q25\% (Hz) & 1270.1 & 30.5 & 2002.7 & 58.4 & 1288.3 & 35.0 & 1754.2 & 58.8 & 1163.3 & 204.4 & 1329.9 & 85.4 \\
\hline Q50\% (Hz) & 2958.8 & 52.1 & 4320.6 & 85.8 & 2759.0 & 62.7 & 3583.1 & 107.5 & 3587.1 & 324.3 & 2439.4 & 221.0 \\
\hline Q75\% (Hz) & 5879.2 & 63.5 & 7141.7 & 88.4 & 5176.9 & 85.2 & 6133.1 & 135.0 & 4684.5 & 350.3 & 4760.1 & 498.6 \\
\hline AmpVar (dB/s) & N/A & N/A & N/A & $\mathrm{N} / \mathrm{A}$ & 199.4 & 4.5 & 183.6 & 6.2 & 165.8 & 17.8 & 125.4 & 10.6 \\
\hline AMRate $\left(\mathrm{s}^{-1}\right)$ & N/A & N/A & N/A & $\mathrm{N} / \mathrm{A}$ & 11.2 & 0.2 & 11.1 & 0.2 & 10.0 & 0.4 & 9.4 & 0.5 \\
\hline AMExtent $(\mathrm{dB})$ & N/A & $\mathrm{N} / \mathrm{A}$ & N/A & N/A & 19.6 & 0.6 & 18.1 & 0.8 & 17.8 & 2.3 & 13.7 & 1.6 \\
\hline Dur (s) & 0.32 & 0.01 & 0.39 & 0.01 & 0.59 & 0.01 & 0.66 & 0.02 & 0.66 & 0.03 & 0.62 & 0.03 \\
\hline
\end{tabular}

\section{Results}

\subsection{Age and sex effects}

As kids matured, their calls became longer, lower in frequency (F0 and formants) and less modulated in frequency and amplitude. Most of these changes were affected by sex, with females having calls with higher frequencies, more frequency modulated and a more stable F0 than males (Tables 1 and 2; Fig. 2; 
see also Fig. 1). Significant age effects indicate general age-related changes, and significant sex effects indicate general sex differences, independent of age. Finally, significant age $\times$ sex effects indicate different age-related changes according to sex.

Table 2 Age and sex effects. Results of the models investigating the effects of age, sex (controlled for age) and the interaction effect $(x)$ between age and sex on the vocal parameters of goat kid calls. The last column indicates R2 values of the full models. See Section 2 for abbreviations of vocal parameters. Bold types indicate significant and marginally significant results $(p<0.07)$.

\begin{tabular}{|c|c|c|c|c|c|c|c|c|c|c|}
\hline \multirow[t]{2}{*}{ Parameter } & \multicolumn{3}{|l|}{ Age } & \multicolumn{3}{|l|}{ Sex } & \multicolumn{3}{|c|}{ Age $x$ Sex } & \multirow{2}{*}{$\frac{\text { Model }}{R^{2}}$} \\
\hline & $d f$ & $\boldsymbol{F}$ & $p$ & $d f$ & $\boldsymbol{F}$ & $p$ & Df & $F$ & $p$ & \\
\hline F0Start $(\mathrm{Hz})$ & 1,1759 & 375.6 & $<.0001$ & 1,10 & 1.2 & 0.30 & 1,1759 & 16.3 & 0.0001 & 0.45 \\
\hline FOEnd $(\mathrm{Hz})$ & 1, 1759 & 732.0 & $<.0001$ & 1,10 & 0.0 & 0.86 & 1,1759 & 68.3 & $<.0001$ & 0.50 \\
\hline FOMean $(\mathrm{Hz})$ & 1,1759 & 811.3 & $<.0001$ & 1,10 & 0.5 & 0.51 & 1,1759 & 55.9 & $<.0001$ & 0.57 \\
\hline FOMin $(\mathrm{Hz})$ & 1,1759 & 561.9 & $<.0001$ & 1,10 & 0.1 & 0.76 & 1,1759 & 70.5 & $<.0001$ & 0.49 \\
\hline FOMax $(\mathrm{Hz})$ & 1,1759 & 933.0 & $<.0001$ & 1,10 & 0.9 & 0.38 & 1,1759 & 25.9 & 0.002 & 0.59 \\
\hline TimeFOMax (\%) & 1,1759 & 3.5 & 0.063 & 1,10 & 0.3 & 0.57 & 1,1759 & 4.2 & 0.042 & 0.03 \\
\hline F0AbsSlope (Hz/s) & 1,1759 & 174.9 & $<.0001$ & 1,10 & 4.8 & 0.053 & 1,1759 & 15.8 & 0.0001 & 0.21 \\
\hline FOVar (Hz/s) & 1,461 & 29.2 & $<.0001$ & 1,10 & 7.3 & 0.022 & 1,461 & 2.9 & 0.089 & 0.13 \\
\hline FMRate $\left(s^{-1}\right)$ & 1,445 & 0.6 & 0.44 & 1,10 & 11.2 & 0.007 & 1,445 & 0.1 & 0.77 & 0.20 \\
\hline FMExtent $(\mathrm{Hz})$ & 1,445 & 22.6 & $<.0001$ & 1,10 & 0.3 & 0.61 & 1,445 & 1.9 & 0.17 & 0.08 \\
\hline Jitter (\%) & 1, 1759 & 0.3 & 0.56 & 1,10 & 6.1 & 0.033 & 1,1759 & 15.2 & 0.0001 & 0.15 \\
\hline Shimmer (\%) & 1,1756 & 0.0 & 0.96 & 1,10 & 7.0 & 0.024 & 1,1756 & 5.5 & 0.019 & 0.22 \\
\hline F1Mean $(\mathrm{Hz})$ & 1,1758 & 163.6 & $<.0001$ & 1,10 & 0.2 & 0.64 & 1,1758 & 4.9 & 0.028 & 0.20 \\
\hline F2Mean $(\mathrm{Hz})$ & 1,1729 & 220.5 & $<.0001$ & 1,10 & 0.0 & 0.97 & 1,1729 & 24.0 & $<.0001$ & 0.24 \\
\hline F3Mean $(\mathrm{Hz})$ & 1,1756 & 1917.6 & $<.0001$ & 1,10 & 4.1 & 0.071 & 1,1756 & 5.5 & 0.019 & 0.53 \\
\hline F4Mean $(\mathrm{Hz})$ & 1,1758 & 2410.4 & $<.0001$ & 1,10 & 3.3 & 0.10 & 1,1758 & 3.9 & 0.049 & 0.58 \\
\hline F1Min $(\mathrm{Hz})$ & 1,1757 & 26.6 & $<.0001$ & 1,10 & 0.3 & 0.58 & 1,1757 & 9.4 & 0.002 & 0.17 \\
\hline F2Min $(\mathrm{Hz})$ & 1, 1729 & 135.6 & $<.0001$ & 1,10 & 0.0 & 0.87 & 1,1729 & 24.3 & $<.0001$ & 0.22 \\
\hline F3Min $(\mathrm{Hz})$ & 1,1756 & 1590.0 & $<.0001$ & 1,10 & 6.4 & 0.030 & 1,1756 & 6.8 & 0.009 & 0.49 \\
\hline F4Min $(\mathrm{Hz})$ & 1,1759 & 1924.5 & $<.0001$ & 1,10 & 5.6 & 0.039 & 1,1759 & 0.4 & 0.52 & 0.53 \\
\hline $\mathrm{F} 1 \mathrm{Max}(\mathrm{Hz})$ & 1,1758 & 220.2 & $<.0001$ & 1,10 & 0.1 & 0.77 & 1,1758 & 3.6 & 0.058 & 0.18 \\
\hline $\mathrm{F} 2 \mathrm{Max}(\mathrm{Hz})$ & 1,1729 & 282.9 & $<.0001$ & 1,10 & 0.0 & 0.99 & 1,1729 & 20.0 & $<.0001$ & 0.23 \\
\hline F3Max (Hz) & 1,1756 & 1363.4 & $<.0001$ & 1,10 & 1.7 & 0.22 & 1,1756 & 5.3 & 0.022 & 0.45 \\
\hline $\mathrm{F} 4 \mathrm{Max}(\mathrm{Hz})$ & 1, 1759 & 2022.6 & $<.0001$ & 1,10 & 1.7 & 0.22 & 1,1759 & 9.5 & 0.002 & 0.54 \\
\hline DfMin (Hz) & 1, 1759 & 1368.5 & $<.0001$ & 1,10 & 3.8 & 0.081 & 1,1759 & 0.2 & 0.66 & 0.47 \\
\hline Q25\% (Hz) & 1, 1759 & 3.2 & 0.076 & 1,10 & 1.2 & 0.29 & 1,1758 & 11.4 & 0.0007 & 0.42 \\
\hline Q50\% (Hz) & 1, 1759 & 4.2 & 0.042 & 1,10 & 2.4 & 0.15 & 1,1759 & 18.0 & $<.0001$ & 0.40 \\
\hline Q75\% (Hz) & 1, 1759 & 113.1 & $<.0001$ & 1,10 & 4.4 & 0.062 & 1,1759 & 4.4 & 0.035 & 0.33 \\
\hline AmpVar (dB/s) & 1,465 & 0.4 & 0.55 & 1,10 & 1.1 & 0.32 & 1,465 & 1.3 & 0.26 & 0.14 \\
\hline AMRate $\left(s^{-1}\right)$ & 1,459 & 12.9 & 0.0004 & 1,10 & 0.0 & 0.98 & 1,459 & 0.2 & 0.62 & 0.11 \\
\hline AMExtent (dB) & 1,459 & 0.7 & 0.40 & 1,10 & 0.7 & 0.43 & 1,459 & 0.1 & 0.71 & 0.14 \\
\hline Dur (s) & 1,1759 & 375.1 & $<.0001$ & 1,10 & 3.9 & 0.077 & 1,1759 & 8.0 & 0.005 & 0.28 \\
\hline
\end{tabular}

There was an effect of age and an interaction effect between age and sex, on all parameters linked to the F0 contour, with F0 values decreasing with age and decreasing faster for males than for females (Tables 1 and 2; see FOMean in Fig. 2). TimeFOMax tended to vary with age $(p=0.063)$ and varied significantly 
differently for males than for females, but the effect was really weak $(R 2=0.03$; Tables 1 and 2$)$. A decrease with age was observed for FOAbsSlope (i.e. F0 slope became less steep). This parameter showed a faster decrease for females than for males (Tables 1 and 2; Fig. 2). The sex effect on F0AbsSlope also tended to be significant $(p=0.053)$, with females having higher F0AbsSlope values than males (Tables 1 and 2; Fig. 2). In the same way, FOvar (cumulative F0 variation) and FMExtent (mean peak-to-peak variation of each F0 modulation) decreased with age (Tables 1 and 2). F0var and FMRate (i.e. number of cycles of FO modulation per second) were higher for females than for males (Tables 1 and 2). Jitter and Shimmer were higher for males than for females. Jitter decreased (i.e. Fo became more stable in frequency) for females but not for males and Shimmer decreased also slightly (i.e. Fo became more stable in amplitude), but the general effect of age was not significant (Tables 1 and 2). To summarise, as kids matured, all the parameters characterising the FO contour of their calls decreased, and decreased faster for males than for females. Several of the measured parameters characterising F0 variation (FOAbsSlope, FOVar, FMExtent) decreased also, indicating a less modulated F0. F0 slope decreased faster for females than for males. Female calls were more frequency modulated (F0var and FMRate) but also had a more stable F0 (Jitter and Shimmer) than male calls.

All formant-related measures decreased with age. They decreased faster for males than for females, except F2- related parameters, which decreased faster for females than for males, and F4Min and DfMin, which decreased in a similar way for males and females (Tables 1 and 2; see FMean in Fig. 2). F3Min and F4Min were generally higher for females than for males (significant sex effect; Tables 1 and 2). Q50\% and Q75\% decreased with age (Tables 1 and 2). All quartiles-related parameters (Q25\%, Q50\% and Q75\%) decreased faster for females than for males, but the general effect of age was not significant for Q25\% (Tables 1 and 2; see Q50\% in Fig. 2). AMRate also decreased (i.e. less cycles of amplitude modulation per second) with age, in the same way for males and females (Tables 1 and 2; Fig. 2). Dur increased with age, and increased faster for males than for females (Tables 1 and 2; Fig. 2). To summarise, as kids matured, all filter-related parameters (except Q25\%) decreased in frequency. The highest measured formants were higher for females than for males throughout development. Calls also became longer in duration and less amplitude modulated.

\subsection{Body size and sex effects}

As kids grew, their calls became lower in frequency (F0 and formants) and had a more stable F0 (in frequency and amplitude). Calls of bigger kids were longer in duration and were composed of a lower number of amplitude modulations, but which were higher (Table 3; Fig. 3). Significant body size effects indicate general body-size related changes with age, and significant sex effects indicate general sex differences, independent of body size.

There was a significant effect of body size on all parameters linked to the F0 contour, except F0Start, with F0 values decreasing when body size increased (Table 3; see F0Min in Fig. 3). F0AbsSlope decreased (i.e. F0 slope became less steep) when body size increased (Table 3; Fig. 3). Jitter and Shimmer decreased (i.e. FO became more stable in frequency and amplitude, respectively) when body size increased (Table 3; Jitter: 8-10 cm, $4.02 \pm 0.18 \%, n=43 ; 10-11.5 \mathrm{~cm}, 3.36 \pm 0.37 \%, \mathrm{n}=20$; for Shimmer, see Fig. 3). The frequencies of the highest measured formants (F3 and F4) and DfMin decreased (i.e. formants became less spaced) when body size increased (Table 3; F3Mean: 8-10 cm, $6481.13 \pm 95.46 \mathrm{~Hz}, \mathrm{n}=43 ; 10-11.5 \mathrm{~cm}, 5924.69 \pm 71.11 \mathrm{~Hz}, \mathrm{n}=20$; F4Mean: 8-10 cm, $8526.84 \pm$ 102.37 Hz, n = 43; 10-11.5 cm, $7812.21 \pm 79.21 \mathrm{~Hz}, \mathrm{n}=20$; for DfMin, see Fig. 3). AMRate decreased (i.e. less cycles of amplitude modulation per second) when body size increased (Table 3; Fig. 3). AMExtend, on the opposite, increased (i.e. higher amplitude modulations) when body size increased (8$10 \mathrm{~cm}, 15.01 \pm 1.47 \mathrm{~dB}, \mathrm{n}=9 ; 10-11.5 \mathrm{~cm}, 19.68 \pm 1.12 \mathrm{~dB}, \mathrm{n}=16$ ). Dur also increased when body size increased (Table 3; Fig. 3). To summarise, as kids grew, all parameters linked to the F0 contour of their 
calls decreased (except F0Start), F0 became less steep (FOAbsSlope) and more stable in frequency and amplitude (Jitter and Shimmer). The highest measured formants and the minimum spacing of the formants decreased, suggesting longer vocal tracts. Calls of bigger kids were also longer in duration and composed of fewer amplitude modulations, but which were higher.

Table 3 Body size and sex effects. Results of the models investigating the effects of body size and sex (controlled for body size) effects on the vocal parameters of goat kid calls. The last column indicates R2 values of the full models. See methods for abbreviations of vocal parameters. Bold types indicate significant and marginally significant results $(p<0.07)$.

\begin{tabular}{|c|c|c|c|c|c|c|c|}
\hline \multirow[t]{2}{*}{ Parameter } & \multicolumn{3}{|c|}{ Body Size } & \multicolumn{3}{|l|}{ Sex } & \multirow{2}{*}{$\begin{array}{r}\text { Model } \\
R^{2}\end{array}$} \\
\hline & $d f$ & $\boldsymbol{F}$ & $p$ & $d f$ & $F$ & $p$ & \\
\hline F0Start (Hz) & 1,39 & 2.3 & 0.14 & 1,10 & 0.5 & 0.50 & 0.33 \\
\hline F0End $(\mathrm{Hz})$ & 1,39 & 12.7 & 0.001 & 1,10 & 0.9 & 0.36 & 0.50 \\
\hline F0Mean (Hz) & 1,39 & 5.4 & 0.025 & 1,10 & 0.0 & 0.90 & 0.42 \\
\hline FOMin $(\mathrm{Hz})$ & 1,39 & 5.2 & 0.028 & 1,10 & 0.0 & 0.85 & 0.50 \\
\hline FOMax $(\mathrm{Hz})$ & 1,39 & 7.2 & 0.011 & 1,10 & 0.2 & 0.67 & 0.41 \\
\hline TimeF0Max (\%) & 1,39 & 0.0 & 0.95 & 1,10 & 1.1 & 0.32 & 0.02 \\
\hline F0AbsSlope (Hz/s) & 1,39 & 28.7 & $<.0001$ & 1,10 & 1.1 & 0.31 & 0.34 \\
\hline FOVar (Hz/s) & 1,6 & 2.4 & 0.17 & 1,8 & 4.7 & 0.063 & 0.27 \\
\hline FMRate $\left(s^{-1}\right)$ & 1,6 & 0.3 & 0.61 & 1,8 & 8.1 & 0.022 & 0.48 \\
\hline FMExtent (Hz) & 1,6 & 3.0 & 0.13 & 1,8 & 1.1 & 0.32 & 0.34 \\
\hline Jitter (\%) & 1,39 & 12.7 & 0.001 & 1,10 & 5.8 & 0.036 & 0.31 \\
\hline Shimmer (\%) & 1,39 & 5.1 & 0.030 & 1,10 & 20.2 & 0.001 & 0.52 \\
\hline F1Mean (Hz) & 1,39 & 2.5 & 0.12 & 1,10 & 2.6 & 0.14 & 0.19 \\
\hline F2Mean $(\mathrm{Hz})$ & 1,39 & 0.8 & 0.37 & 1,10 & 1.1 & 0.32 & 0.18 \\
\hline F3Mean (Hz) & 1,39 & 47.0 & $<.0001$ & 1,10 & 2.0 & 0.18 & 0.39 \\
\hline F4Mean (Hz) & 1,39 & 56.6 & $<.0001$ & 1,10 & 1.5 & 0.25 & 0.39 \\
\hline F1Min (Hz) & 1,39 & 3.0 & 0.092 & 1,10 & 1.4 & 0.26 & 0.21 \\
\hline F2Min (Hz) & 1,39 & 0.7 & 0.40 & 1,10 & 3.6 & 0.088 & 0.22 \\
\hline F3Min (Hz) & 1,39 & 41.8 & $<.0001$ & 1,10 & 2.1 & 0.18 & 0.34 \\
\hline F4Min (Hz) & 1,39 & 53.7 & $<.0001$ & 1,10 & 2.3 & 0.16 & 0.38 \\
\hline F1Max $(\mathrm{Hz})$ & 1,39 & 2.7 & 0.11 & 1,10 & 0.9 & 0.36 & 0.20 \\
\hline $\mathrm{F} 2 \mathrm{Max}(\mathrm{Hz})$ & 1,39 & 2.9 & 0.098 & 1,10 & 0.8 & 0.40 & 0.19 \\
\hline F3Max $(\mathrm{Hz})$ & 1,39 & 41.4 & $<.0001$ & 1,10 & 1.5 & 0.25 & 0.41 \\
\hline F4Max $(\mathrm{Hz})$ & 1,39 & 58.2 & $<.0001$ & 1,10 & 0.7 & 0.43 & 0.38 \\
\hline DfMin (Hz) & 1,39 & 57.4 & $<.0001$ & 1,10 & 0.6 & 0.46 & 0.39 \\
\hline Q25\% (Hz) & 1,39 & 1.9 & 0.17 & 1,10 & 21.1 & 0.001 & 0.46 \\
\hline Q50\% (Hz) & 1,39 & 0.0 & 1.00 & 1,10 & 20.4 & 0.001 & 0.43 \\
\hline Q75\% (Hz) & 1,39 & 0.6 & 0.44 & 1,10 & 5.7 & 0.038 & 0.29 \\
\hline AmpVar (dB/s) & 1,6 & 1.4 & 0.28 & 1,8 & 0.0 & 0.95 & 0.06 \\
\hline AMRate $\left(\mathrm{s}^{-1}\right)$ & 1,6 & 12.4 & 0.013 & 1,8 & 0.7 & 0.42 & 0.44 \\
\hline AMExtent (dB) & 1,6 & 10.5 & 0.018 & 1,8 & 0.4 & 0.54 & 0.33 \\
\hline Dur (s) & 1,39 & 24.4 & $<.0001$ & 1,10 & 0.6 & 0.44 & 0.33 \\
\hline
\end{tabular}



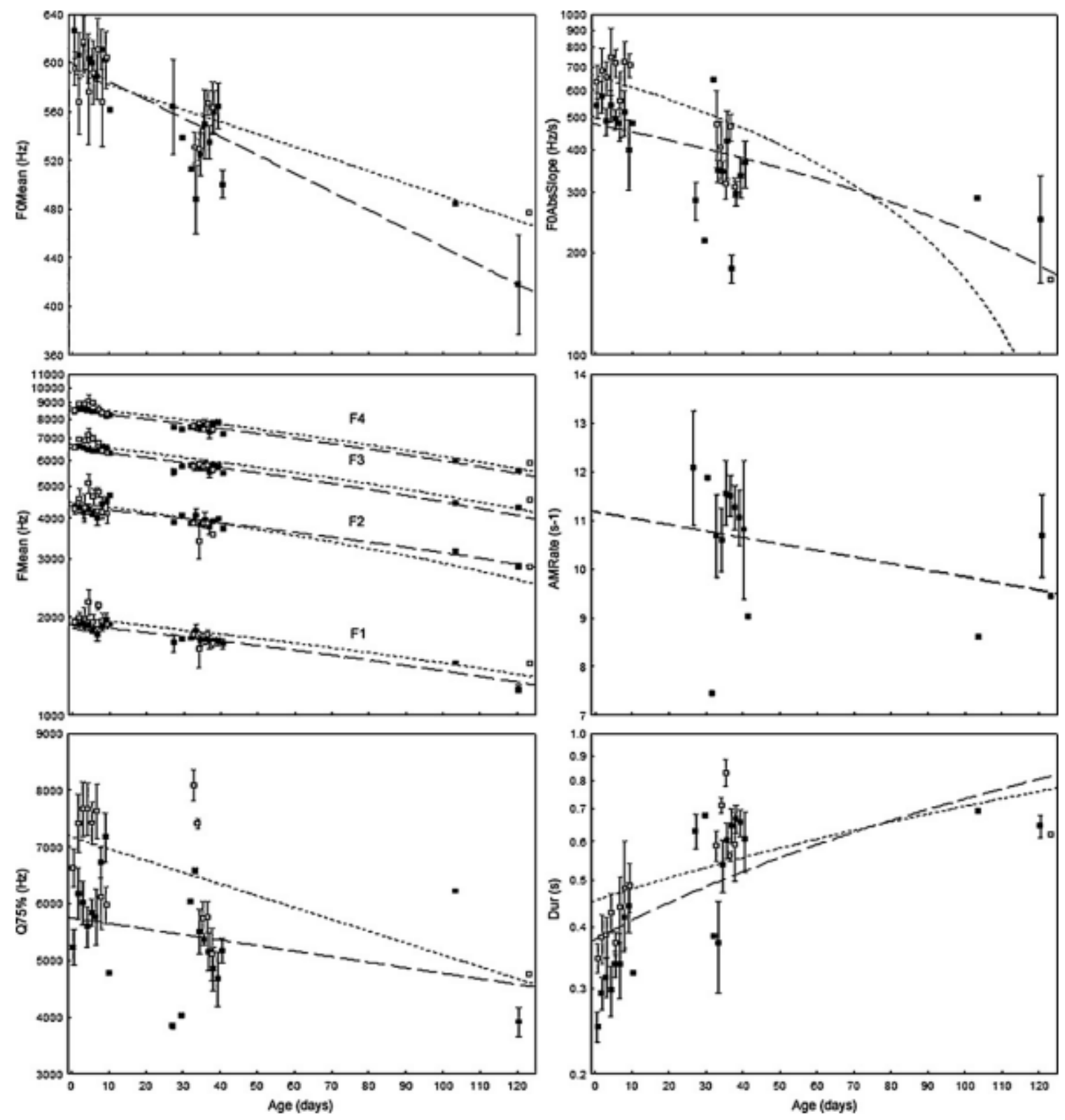

Fig. 2. Age effects on vocal parameters. Mean F0 (FOMean), F0 abosolute slope (FOAbsSlope, log-transformed), mean frequency of the first four formants (F1-4Mean, log-transformed), amplitude modulation rate (AMRate), frequency of the third quartiles of energy $(\mathrm{Q} 75 \%)$ and call duration (Dur, log-transformed) in relation to age (mean \pm SE per day). Lines indicate the best fits. When the effect of sex or of the interaction between sex and age was significant, female values are indicated with empty squares and dotted lines and male values with full squares and dashed lines.

\section{Discussion}

To our knowledge, our study is the first to describe in detail how age and body size growth are linked to changes in the structure of calls according to sex in mammals (other than humans) from a source-filter theory perspective (i.e. investigating changes in both source- and filter-related parameters; Fant, 1960). We investigated age and body size-related modifications to the vocal characteristics during ontogeny in goat kids, by following the same individuals from birth to four months old. We found age-related changes 
to most of the vocal parameters measured and almost all of these changes were sex-dependent. As kids matured, their calls were longer in duration, had lower frequencies ( $F 0$ and formants), and were less frequency and amplitude modulated. With age, female calls became higher in frequency than male calls. Female calls were also more frequency modulated and had a more stable fundamental frequency (F0) than male calls. Body size growth was responsible for several of these changes, including changes in F0 and changes in the frequencies of the highest measured formants (third and fourth formants). Therefore, kid calls provide important information about age, sex and body size.

\subsection{Changes with age according to sex}

We found that most of the vocal parameters changed with age, and this also depended on sex. These variations between male and female calls are likely due to differences in sex hormones affecting the vocal production organ either directly (Charlton et al., 2011), or indirectly by affecting other physical characteristics (e.g. body growth), which subsequently affect the vocal organ (Beckford et al., 1985). We found that FO contour, most formants and call duration changed with age in a different way for males and females. Because most of these parameters are also linked to body size changes, these differences probably result from sex hormones causing differing male and female body size growth (indirect effect of hormones on the vocal apparatus). Age-related changes to mean F0 and call duration according to sex described in our study are very similar to what has been found in both harbour seals (Phoca vitulina richardii, Khan and Markowitz, 2006) and chacma baboons (Ey et al., 2007b). The mean F0 decreased with age in both sexes, but became higher in females than in males, and call duration was longer in females than males during the first days and became shorter later. This suggests that some similar mechanisms exist across mammals. When they grow, males become larger than females, and therefore should have larger lung capacity and longer vocal folds. This would enable them to produce longer calls with lower F0. However, the longer duration calls produced by young female goats (this study), harbour seals (Khan and Markowitz, 2006) and chacma baboons (Ey et al., 2007b), despite an overall smaller size and weight than males must be due to other mechanisms, such as sex-linked behavioural differences, or potentially different reactions of mothers towards male and female offspring.

Sex differences in age-related changes to vocal parameters that are independent of body size may result from different effects of male and female sex hormones on the vocal production organ during development (direct effect of hormones on the vocal apparatus). In humans, the lowest formants (F1 and F2) are determined by the length, width and volume of the vocal tract cavity resonances and also on some characteristics of the vocal tract walls (hard and soft tissues, Titze, 1994; Fitch and Hauser, 2002). In goats, F2 is absent or reduced in closed-mouth contact calls produced when individuals are close to each other (EB, personal observation), which suggests that F2 depends mostly on the opening of the mouth. Observed sex differences in the lowest formants and frequency values of energy quartiles are thus probably linked to different age-related changes, which are independent of body size, in the width and volume of the vocal tract cavities between males and females.

\subsection{Body-size related changes}

We demonstrated negative relationships between most frequency parameters and body size during growth. Body size growth influenced both source- (F0) and filter-related frequencies (formants), and also vocal parameters linked to the respiratory system (amplitude and duration). F0 contour is determined by the anatomy/physiology of the larynx and the sub-laryngeal and laryngeal structures. The larynx is not constrained by neighbouring skeletal structures, so that vocal fold length is not precisely dependent on body size. However, F0 can be correlated with body size because of age-related vocal folds growth (Taylor and Reby, 2010). Body size-related lowering of F0-contour parameters in our study thus probably results from a lengthening of vocal folds with age. Formant frequencies are determined by the 
anatomy/physiology of the supralaryngeal vocal tract. Contrary to F0, the vocal tract is constrained by the bones of the skull, jaw, and spinal column, so that its length strongly depends on body size (Fitch and Giedd, 1999). The minimum spacing of the formants (DfMin) is a good cue to caller size in several species (Reby and McComb, 2003; Vannoni and McElligott, 2008; Charlton et al., 2009b). Our study shows that this parameter also decreases with age because of body size growth.
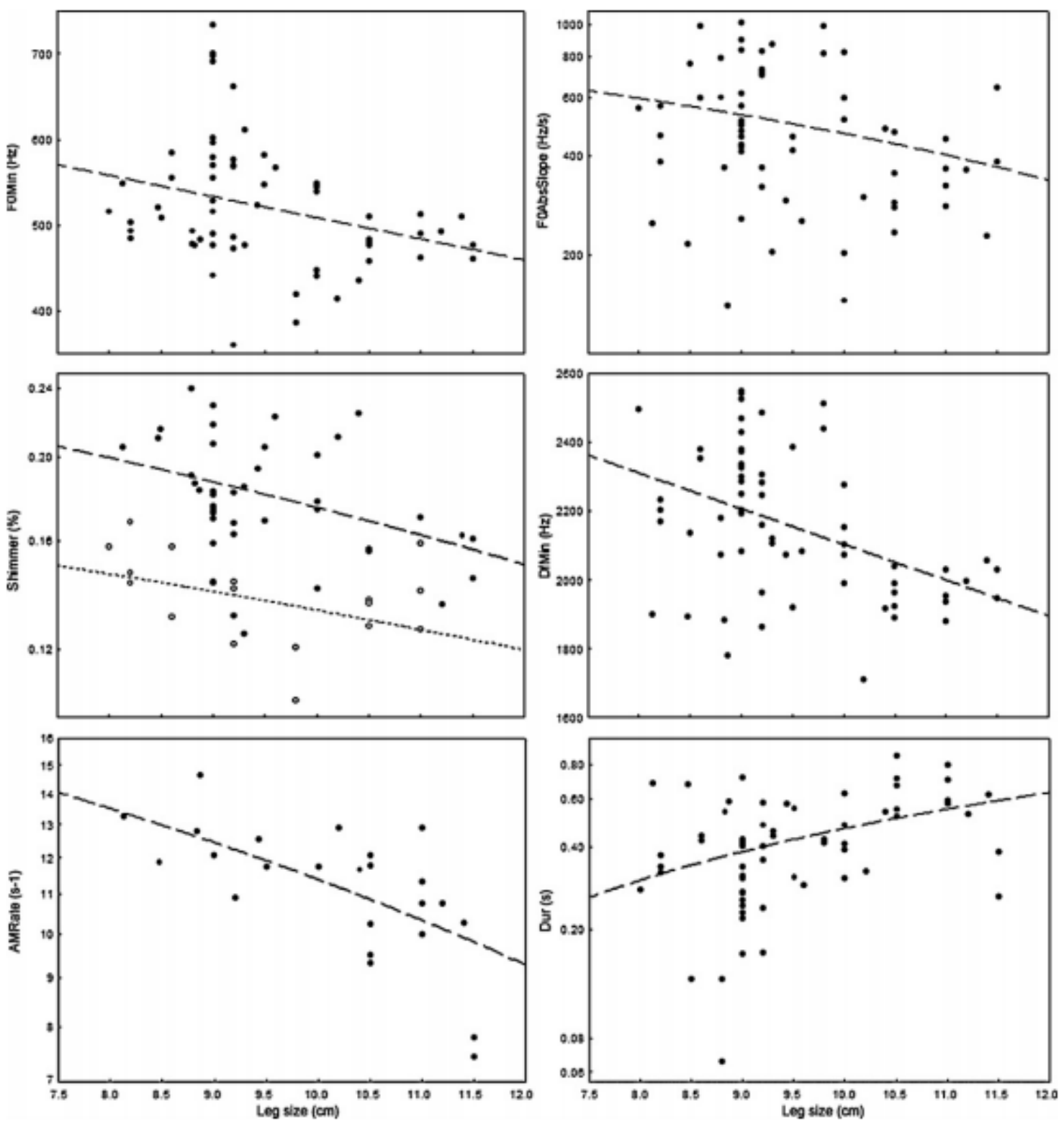

Fig. 3. Body size effects on vocal parameters. Minimum F0 (FOMean), F0 abosolute slope (F0AbsSlope, log-transformed), Shimmer (log-transformed), minimum spacing of the formants (DfMin), amplitude modulation rate (AMRate, log-transformed), and call duration (Dur, log-transformed) in relation to leg size (scatterplot). Lines indicate the best fits. When the effect of sex or of the interaction between sex and age was significant, female values are indicated with empty circles and dotted lines and male values with full circles and dashed lines. Dots indicate the mean values of vocal parameters per kid per day ( $n=8$ calls per day). 
Higher formants depend more directly on the vocal tract length than lower formants, and are therefore expected to be more precisely related to body size than lower formants (Fant, 1960; Fitch and Hauser, 2002). This is con- firmed in our study by body size-related changes to the highest measured formants (F3 and F4) and not to the lowest measured formants (F1 and F2). Finally, duration and amplitude modulations are determined by coordination between the respiratory system and the source (changes in airflow or sub-glottal pressure, Titze, 1994). We found that calls became longer in duration and with a lower number of amplitude modulations, but which were higher. These changes can be explained by a positive correlation between lung capacity and body size, resulting in individuals with larger lungs producing longer and louder calls (Fitch and Hauser, 2002), which is often observed among mammals (Torriani et al., 2006; Ey et al., 2007a).

All male kids in our study were castrated by applying a constrictive rubber ring (elastors) to the neck of the scrotum 3-4 days after birth. This method causes the testicles to fall off after 10-14 days. Early castration results in a slightly lower growth rate compared to intact male kids, but higher than for female kids (Louca et al., 1977). These growth differences and the differences in vocal parameters found in our study between castrated males and females suggest an important role of prenatal testosterone on castrated male development. Differences between calls of intact male kids and female kids might in fact be higher than between castrated male kids and female kids. However, because testosterone levels in male goats are very low before sexual maturity (i.e. 22 weeks/154 days on average; Chakraborty et al., 1989; Ahmad and Noakes, 1996), these differences between castrated and intact males are likely to have had little impact on the goats used in our study (0-124 days old). Castration can also have an indirect effect on vocalisations by causing pain. In pigs (Sus scrofa), pain associated with removing the testicles with a scalpel, was shown to increase the number of high frequency calls produced (Weary et al., 1998). Constrictive rubber rings cause some minor discomfort during the first hours following the procedure, with plasma cortisol levels returning to pretreatment values within approximately $2 \mathrm{~h}$ (Mellor et al., 1991; Kent et al., 1993). Potential pain-related changes in the vocalisations of males in our study should thus be visible around 3-5 days postpartum. According to Fig. 2, such changes do not occur. An increase in the frequencies of the energy quartiles and of the formants seems to happen around days 9 and 10 (Fig. 2), but these changes are likely due to factors other than the discomfort caused by the rubber ring (applied at days 3-4).

\section{Conclusion}

We showed that goat kid vocalisations provide important information, which is potentially available to others in their social groups, related to individuality (Briefer and McElligott, in press), age, sex and body size. The study of vocalisations from a source-filter theory perspective can provide vital information about an animal's physical characteristics (see Taylor and Reby, 2010). This framework is also likely to reveal important structural differences between calls, which reflect affective states of various arousal levels and valence (positive/negative). We recommend that the framework should be adopted in other applied studies of animal vocalisations because it will greatly assist progress.

\section{Acknowledgments}

We are grateful to E. Antill, C. Booth, E. Cant, C. Charpin, K. Cho Geun-A, C. Farrington, F. Galbraith, L. Kashap, J. Kemp, E. Landy, M. Padilla de la Torre and M. Wang for assistance, and to two anonymous referees for helpful comments on the manuscript. We thank $D$. Reby for providing the custom built program in Praat. E. Briefer is funded by a Swiss National Science Foundation fellowship. We acknowledge the financial support of the University of London Central Research Fund. We thank the staff of White Post Farm (http://whitepostfarmcentre.co.uk/) for their help and free access to the animals. 


\section{References}

Ahmad, N., Noakes, D.E., 1996. Sexual maturity in British breeds of goat kids. Br. Vet. J. 152, 93-103.

Aufdemorte, T.B., Sheridan, P.J., Holt, G.R., 1993. Autoradiographic evidence of sex steroid receptors in aryngealtissues of the baboon (Papio cynocephalus). Laryngoscope 93, 1607-1611.

Beckford, N.S.,Rood, S.R., Schaid, D., Schanbacher, B., 1985.Androgen stimulation and laryngeal development. Ann. Otol. Rhinol. Laryngol. 94, 634-640.

Boersma, P., Weenink, D., 2009. Praat: doing phonetics by computer. http://www.praat.org/. Briefer, E., McElligott, A.G. Mutual mother-offspring vocal recognition in an ungulate hider species (Capra hircus). Anim. Cogn., in press.

Briefer, E.,Vannoni, E., McElligott,A.G., 2010. Quality prevails over identity in the sexually selected vocalisations of an ageing mammal. BMC Biol. 8, 35.

Chakraborty, P.K., Stuart, L.D.,Brown,J.L., 1989. Puberty inthemaleNubian Goat: serum concentrations of $\mathrm{LH}, \mathrm{FSH}$ and testosterone from birth through puberty and semen characteristics at sexual maturity. Anim. Reprod. Sci. 20, 91-101.

Charlton, B.D., Zhihe, Z., Snyder, R.J., 2009a. Vocal cues to identity and relatedness in giant pandas (Ailuropoda melanoleuca). J. Acoust. Soc. Am. 126, 2721-2732.

Charlton, B.D., Zhihe, Z., Snyder, R.J., 2009b. The information content of giant panda, Ailuropoda melanoleuca, bleats: acoustic cues to sex, age and size. Anim. Behav. 78, 893-898.

Charlton, B.D., Keating, J.L., Rengui, L., Huang, Y., Swaisgood, R.R., 2010. Female giant panda (Ailuropoda melanoleuca) chirps advertise the caller's fertile phase. Proc. R. Soc. B 277, 11011106.

Charlton, B.D., Keating, J.L., Kersey, D., Rengui, L., Huang, Y., Swaisgood, R.R., 2011. Vocal cues to male androgen levels in giant pandas. Biol. Lett. 7, 71-74.

Coltman, D.W., Smith, J.A., Bancroft, D.R., Pilkington, J., MacColl, A.D.C., et al., 1999. Densitydependent variation in lifetime breeding success and natural and sexual selection in Soay rams. Am. Nat. 154, 730-746.

Defra, 2009.Code of Recommendations for the Welfare of Livestock: Goats. Defra, London. Ey, E., Pfefferle, D., Fischer, J., 2007a. Do age- and sex-related variations reliably reflect body size in non-human primate vocalizations? A review. Primates 48, 253-267.

Ey, E., Hammerschmidt, K., Seyfarth, R.M., Fisher, J., 2007b. Age- and sexrelated variations in clear calls of Papio ursinus. Int. J. Primatol. 28, 947-960.

Fant, G., 1960. Acoustic Theory of Speech Production. Mouton, The Hague.

Fitch, W.T., Hauser, M.D., 2002. Unpacking "honesty": vertebrate vocal production and the evolution of acoustic signals. In:Megala-Simmons, A., Popper, A.(Eds.), Acoustic Communication. SpringerVerlag, Berlin, pp. 65-137.

Fitch, W.T., Giedd, J., 1999. Morphology and development of the human vocal tract: a study using magnetic resonance imaging. J. Acoust. Soc. Am. 106, 1511-1522. 
Gogoleva, S., Svetlana, S., Volodin, I., Volodina, E., Kharlamova, A., Trut, L.N., 2010. Sign and strength of emotional arousal: vocal correlates of positive and negative attitudes to humans in silver foxes (Vulpes vulpes). Behaviour 147, 1713-1736.

Hollén, L.I., Clutton-Brock, T., Manser, M.B., 2008. Ontogenetic changes in alarm-call production and usage in meerkats (Suricata suricatta): adaptations or constraints? Behav. Ecol. Sociobiol. 62, 821-829.

Janik, V.M., Slater, P.J.B., 1997. Vocal learning in mammals. Adv. Stud. Behav. 26, 59-99.

Jones, G., Ransome, R.D., 1993. Echolocation calls of bats are influenced by maternal effects and change over a lifetime. Proc. R. Soc. B 252, 125-128.

Kent, J.E., Molony, V., Robertson, I.S., 1993. Changes in plasma cortisol concentration in lambs of three ages after three methods of castration and tail docking. Res. Vet. Sci. 55, 246-251.

Khan, C.B., Markowitz, H., 2006. Vocal development in captive harbor seal pups, Phoca vitulina richardii: age, sex, and individual differences. J. Acoust. Soc. Am. 120, 1684-1694.

Lenhardt, M.L., 1977. Vocal contour cues in maternal recognition of goat kids. Appl. Anim. Ethol. 3, 211219.

Louca, A., Economides, S., Hancock, J., 1977. Effects of castration on growth rate, feed conversion efficiency and carcass quality in Damascus goats. Anim. Prod. 24, 387-391.

Magee, L., 1990. R2 measures based on Wald and likelihood ratio joint significance tests. Am. Stat. 44, 250-253.

Manteuffel, G., Puppe, B., Schön, P.C., 2004. Vocalization of farm animals as a measure of welfare. Appl. Anim. Behav. Sci. 88, 163-182.

McElligott, A.G., Gammell, M.P., Harty, H.C., Paini, D.R., Murphy, D.T., Walsh, J.T., Hayden, T.J., 2001. Sexual size dimorphism in fallow deer (Dama dama): do larger, heavier males gain greater mating success? Behav. Ecol. Sociobiol. 49, 266-272.

Mellor, D.J., Molony, V., Robertson, I.S., 1991. Effects of castration on behaviour and plasma cortisol concentrations in young lambs, kids and calves. Res. Vet. Sci. 51, 149-154.

Miranda-de la Lama, G.C., Mattiello, S., 2010. The importance of social behaviour for goat welfare in livestock farming. Small Ruminant Res. 90, 1-10.

Peters, G., 2011. Dominant frequency of loud mew calls of felids (Mammalia: Carnivora) decreases during ontogenetic growth. Mammal Rev. 41, 54-74.

Poindron, P., Nowak, R., Levy, F., Porter, R.H., Schaal, B., 1993. Development of exclusive motheryoung bonding in sheep and goats. Oxf. Rev. Reprod. Biol. 15, 311-364.

R Development Core Team, 2009. R Foundation for Statistical Computing. Vienna, Austria. http://www.Rproject.org.

Reby, D., Cargnelutti, B., Hewison,A.J.M., 1999. Contexts and possible functions of barking in roe deer. Anim. Behav. 57, 1121-1128. 
Reby, D., McComb, K., 2003. Anatomical constraints generate honesty: acoustic cues to age and weight in the roars of red deer stags. Anim. Behav. 65, 519-530.

Reby, D., McComb, K., Cargnelutti, B., Darwin, C., Fitch, W., Clutton-Brock, T., 2005. Red deer stags use formants as assessment cues during intrasexual agonistic interactions. Proc. R. Soc. B 272, 941947.

Rendall, D., Notman, H., Owren, M.J., 2009. Asymmetries in the individual distinctiveness and maternal recognition of infant contact calls and distress screams in baboons. J. Acoust. Soc. Am. 125, 1792-1805.

Ruiz-Miranda, C., Szymanski, M., Ingals, J., 1993. Physical characteristics of the vocalizations of domestic goat does Capra hircus in response to their offspring's cries. Bioacoustics 5, 99-116.

Sèbe, F., Duboscq, J., Aubin, T., Ligout, S., Poindron, P., 2010. Early vocal recognition of mother by lambs: contribution of low- and highfrequency vocalizations. Anim. Behav. 79, 1055-1066.

Scherer, K.R., 1986. Vocal affect expression: a review and a model for future research. Psychol. Bull. 99, 143-165.

Seyfarth, R.M., Cheney, D.L., 2010. Production, usage, and comprehension in animal vocalizations. Brain Lang. 115, 92-100.

Sueur, J., Aubin, T., Simonis, C., 2008. Equipment Review: Seewave, a free modular tool for sound analysis and synthesis. Bioacoustics 18, 213-226.

Taylor, A.M., Reby, D., 2010. The contribution of source-filter theory to mammal vocal communication research. J. Zool. 280, 221-236.

Titze, I.R., Horii, Y., Scherer, R., 1987. Some technical considerations in voice perturbation measurements. J. Speech Lang. Hear. Res. 30, 252-260.

Titze, I., 1994. Principles of Voice Production. Prentice Hall, Englewood Cliffs, NJ.

Torriani, V.G., Vannoni, E., McElligott, A.G., 2006. Mother-young recognition in an ungulate hider species: a unidirectional process. Am. Nat. 168, 412-420.

Vanková, D., Málek, J., 1997. Characteristics of the vocalisations of Red Deer Cervus elaphus hinds and calves. Bioacoustics 7, 281-289.

Vannoni, E., McElligott, A.G., 2007. Individual acoustic variation in fallow deer (Dama dama) common and harsh groans: a source-filter theory perspective. Ethology 113, 223-234.

Vannoni, E., McElligott, A.G., 2008. Low frequency groans indicate larger and more dominant fallow deer (Dama dama) males. PLoS One 3, e3113.

Venables, R., Ripley, B., 2001. Modern Applied Statistics with S. Allyn and Bacon, New York.

Weary, D.M., Braithwaite, L.A., Fraser, D., 1998. Vocal response to pain in piglets. Appl. Anim. Behav. Sci. 56, 161-172.

Weary, D.M., Fraser, D., 1995. Signalling need: costly signals and animal welfare assessment. Appl. Anim. Behav. Sci. 44, 159-169. 